\title{
CONDIÇÃO DE SAÚDE AUTO PERCEBIDA E PREVALÊNCIA DE DOENÇAS CRÔNICAS NÃO TRANSMISSÍVEIS EM IDOSOS ATENDIDOS PELA ESTRATÉGIA DA SAÚDE DA FAMÍLIA
}

\author{
AUTO PERCEIVED HEALTH CONDITION AND PREVALENCE OF CHRONIC DISEASES NON- \\ TRANSMISSIBLE IN ELDERLY FAMILY HEALTH STRATEGY
}

Flavia Kirsch Dresch'; Anna Regina Grings Barcelos²
Gilson Luis da Cunha 3 . Geraldine Alves dos Santos 4

Recebido em: 26 de abril de 2017

Aprovado em: 09 de junho de 2017

Sistema de Avaliação: Double Blind Review

RCO | a. 9 | v. 2 | p. $118-127$ | jul./dez. 2017

\begin{abstract}
RESUMO
O presente estudo teve como objetivo identificar a condição de saúde auto percebida e a prevalência de doenças crônicas não transmissíveis em idosos atendidos pela Estratégia da Saúde da Família de um município da Região do Vale do Rio dos Sinos, RS. O método de pesquisa foi de natureza quantitativa com abordagem descritiva, de corte transversal. A amostra do estudo foi constituída de 50 idosos com variação de idade de 60 a 91 anos, composta por 66\% de mulheres e 52\% estão na faixa dos 60 aos 69 anos. O instrumento utilizado para a coleta de dados foi um questionário contendo informações de auto percepção de saúde e prática de atividade física. Para a análise dos dados utilizou-se a estatística descritiva através das distribuições de frequências absolutas (n) e relativas (\%). Os resultados demonstraram que $72 \%$ dos idosos avaliaram seu estado de saúde como sendo positivo. A doença crônica não transmissível de maior prevalência foi a Hipertensão Arterial (64\%), seguida pelas doenças osteoarticulares (artrite/reumatismo/artrose), com 36\%. As dislipidemias foram citadas por 34\% dos entrevistados e a diabetes por $28 \%$. Considerando os resultados expostos, percebe-se que os idosos têm uma percepção positiva da sua saúde, mesmo convivendo com a presença de doenças crônicas não transmissíveis.
\end{abstract}

Palavras-chave: Auto percepção de Saúde. Doenças Crônicas não Transmissíveis. Envelhecimento.

\begin{abstract}
The present study aimed to identify the self - perceived health condition and the prevalence of chronic non - transmissible diseases in the elderly attended by the Family Health Strategy of a city in the Vale do Rio dos Sinos/RS. The research method was of a quantitative design with a descriptive and crosssectional approach. For the collection of data was used a questionnaire containing information about self - perception of health and practice of physical activity. For the analysis of the data, the descriptive statistics were used through the distributions of absolute frequencies (n) and relative frequencies (\%). The study sample consisted of 50 elderly people with 60 to 91 years, composed of $66 \%$ of women and $52 \%$ of 60 to 69 years. It was observed that $72 \%$ of the elderly evaluated their positive health status. The most prevalent non-transmissible chronic disease was Arterial Hypertension (64\%), followed by

\footnotetext{
${ }^{1}$ Graduada em Educação Física pela Universidade Feevale (Novo Hamburgo/Brasil). E-mail: flavinhakd@ gmail.com.

${ }^{2}$ Especialista em Educação Física para Terceira Idade pela Universidade do Vale do Rio dos Sinos (São Leopoldo/Brasil). Email: anna@e-abs.com.br.

${ }^{3}$ Doutor em Genética e Biologia Molecular pela Universidade Federal do Rio Grande do Sul (Porto Alegre/Brasil). Professor no Instituto de Administração Hospitalar e Ciências da Saúde (Porto Alegre/Brasil). E-mail: gilsonlcunha@ feevale.br.

${ }^{4}$ Doutora em Psicologia pela Pontifícia Universidade Católica do Rio Grande do Sul (Porto Alegre/Brasil). Professora na Universidade Feevale (Novo Hamburgo/Brasil). E-mail: geraldinesantos@ feevale.br.
} 
osteoarticular diseases (arthritis / rheumatism / arthrosis), with 36\%. Dyslipidemias were cited by $34 \%$ of respondents and diabetes by $28 \%$. Considering the aforementioned results, it was observed that the elderly have a positive perception of their health, even living with the presence of chronic nontransmissible diseases.

Keywords: Self-perception of health. Chronic non-transmissible diseases. Aging.

\section{INTRODUÇÃO}

O Brasil tem vivenciado importantes mudanças no perfil demográfico e na estrutura etária populacional com elevação da expectativa de vida e acentuado envelhecimento da população. Com o avanço das tecnologias da área da saúde, valorização da atenção primária à saúde, desenvolvimento de políticas públicas saudáveis voltadas aos idosos e o novo paradigma da promoção da saúde, dentre outros fatores, houve contribuição para que os indivíduos envelheçam saudáveis, atingindo idade cada vez mais avançada (PILGER; MENON; MATHIAS, 2011).

Desta forma, o acelerado envelhecimento da população, tanto em países desenvolvidos quanto em desenvolvimento, tem levado a uma busca por indicadores de condição de saúde, que possam ser usados tanto em inquéritos de saúde quanto em estudos etiológicos. Com o aumento geral da sobrevida da população, ressalta-se a importância de garantir aos idosos não apenas maior longevidade, mas felicidade, qualidade de vida e satisfação pessoal. Diante deste contexto, o envelhecimento da população mundial representa um trunfo para a humanidade, porém traz um desafio social de estruturação para o atendimento das necessidades desse grupo.

Neste sentido, a condição de saúde percebida ou auto percepção de saúde é considerada importante preditor de incapacidade funcional nos idosos. A presença de problemas físicos, psíquicos, emocionais e sociais acaba gerando sentimentos de fragilidade e insegurança, refletindo de forma negativa no desempenho das funções. Assim, a auto percepção de saúde vem sendo considerada um método confiável utilizado na abordagem da associação entre saúde, determinantes demográficos e socioeconômicos, doenças crônicas e capacidade funcional em idosos (PITANGA, 2010).

No que refere aos aspectos conceituais, Pitanga (2010) comenta que a saúde pode ser dividida em saúde positiva ou negativa associada à condição humana representada por dimensões física, social e psicológica. A saúde positiva estaria relacionada à capacidade de apreciar a vida e de superar os desafios do cotidiano, e a saúde negativa estaria associada à morbidade e a mortalidade.

O envelhecimento humano faz parte de um conjunto de alterações morfofuncionais que levam a uma desestruturação orgânica, contínua e irreversível no organismo. Segundo Terra, Oppermann e Terra (2010) o envelhecimento é um processo dinâmico e progressivo. Ele provoca no organismo modificações biológicas, psicológicas e sociais e é na velhice que este processo aparece de forma mais evidente. As modificações biológicas são as morfológicas, reveladas por aparecimento de rugas, cabelos brancos e outras; fisiológicas, relacionadas às alterações das funções orgânicas; e bioquímicas, que estão diretamente ligadas às transformações das reações químicas que se processam no organismo. As modificações psicológicas ocorrem quando, ao envelhecer, o ser humano precisa adaptar-se a cada situação nova do seu cotidiano. As modificações sociais são verificadas quando as relações sociais tornam-se alteradas em função da diminuição da produtividade e, principalmente, do poder físico e econômico, sendo a alteração social mais evidente em países de economia capitalista (SANTOS, 2010).

A velhice é um processo complexo de alterações na trajetória de vida das pessoas. Cada contexto tem particularidades que vão alterar o estilo de vidas de cada um, com isso, os modos de revelar o 
significado da velhice e do processo de envelhecer para os idosos dependem de como viveu essa pessoa e como as adaptações e enfrentamentos cotidianos são feitos. Repercussão do envelhecer é respondida por eles de maneira diferente, dependendo da história de vida pessoal, da disponibilidade de suporte afetivo, das redes sociais, do sistema de valores pessoais e do estilo de vida adotado por cada um (FREITAS; QUEIROZ; SOUSA, 2010).

Compreende-se, portanto, que o envelhecimento, segundo Souza (2010, p. 26) "é um processo complexo, individual e que envolve diversas variáveis; predisposição genética, estilo de vida, doenças crônicas, entre outras, que interagem entre si e influenciam significativamente a maneira pela qual o indivíduo atinge determinada idade".

Ressaltando o fator idade, a maioria das doenças crônicas que acometem o indivíduo idoso tem na própria idade seu principal fator de risco. Envelhecer sem nenhuma doença crônica é mais uma exceção do que uma regra (BRASIL, 2011a). Conforme as pessoas envelhecem, as doenças não transmissíveis (DCNT) transformam-se nas principais causas de morbidade, incapacidade e mortalidade em todas as regiões do mundo, correspondendo a $63 \%$ dos óbitos em 2008. Aproximadamente $80 \%$ das mortes por DCNT ocorrem em países de baixa e média renda. Um terço dessas mortes ocorre em pessoas com idade inferior a 60 anos. A maioria dos óbitos por DCNT são atribuíveis às doenças do aparelho circulatório (DAC), ao câncer, à diabetes e às doenças respiratórias crônicas. As principais causas dessas doenças incluem fatores de risco modificáveis, como tabagismo, consumo nocivo de bebida alcoólica, inatividade física e alimentação inadequada (BRASIL 2011c).

Camarano (2014, p. 644) reconhece sobre o avanço nos estudos associados a fatores de risco que:

[...] ainda há pouco conhecimento acumulado sobre as causas de várias doenças crônicas, embora tenha havido avanços no estudo dos fatores de risco. Entre esses fatores, consideram-se: o tabagismo, a alimentação inadequada, o álcool e a inatividade física. Os elementos explicativos estão associados a fatores não modificáveis, como a genética, e a determinantes macroeconômicos e sociais. Os fatores de risco intermediários mais importantes estão relacionados a hipertensão, dislipidemia, obesidade/sobrepeso e intolerância à glicose.

A Hipertensão Arterial é a doença crônica não transmissível mais predominante entre os idosos. Sua prevalência aumenta progressivamente com o envelhecimento, sendo considerada o principal fator de risco cardiovascular modificável na população geriátrica. A HAS frequentemente se associa a distúrbios metabólicos, alterações funcionais e/ou estruturais de órgãos-alvo, sendo agravada pela presença de outros fatores de risco, como dislipidemia, obesidade abdominal, intolerância à glicose e diabete mellitus (MALACHIAS et al., 2016).

Conforme Brasil (2011c) as doenças crônicas não transmissíveis constituem o problema de saúde de maior magnitude e correspondem a 72\% das causas de mortes. Em 2007, a taxa de mortalidade por DCNT no Brasil foi de 540 óbitos por 100 mil habitantes (SCHMIDT, 2011). Apesar de elevada, observou-se redução de $20 \%$ nessa taxa na última década, principalmente em relação às doenças do aparelho circulatório e respiratórias crônicas. Entretanto, as taxas de mortalidade por diabetes e câncer aumentaram nesse mesmo período. A redução das DCNT pode ser, em parte, atribuída à expansão da Atenção Básica, melhoria da assistência e redução do tabagismo nas últimas duas décadas, que passou de $34,8 \%$ (1989) para $15,1 \%$ (2010).

Prever o impacto do envelhecimento no aumento da incidência de doenças e consequentemente, nos gastos com saúde, conforme Camarano (2014) é dificultado pelo desconhecimento da evolução de padrões de comportamento, como cuidados nutricionais, prática de atividades físicas e redução ou 
suspensão do consumo de tabaco, bem como por políticas de promoção e prevenção à saúde. Para Pitanga (2010) isso significa adquirir novos hábitos, aceitar mudanças, modificar o estilo de vida prejudicial à saúde, estabelecer relações sociais e familiares positivas e consistentes, fatores que contribuem para o aumento da qualidade de vida, indispensável para um envelhecimento saudável.

Camarano (2014, p. 645) não tem dúvida ao afirmar que:

O foco do sistema de saúde deverá ser na combinação de ações de promoção e prevenção, mais relacionadas aos processos de cronicidade das doenças, paralelamente ao desenvolvimento de ações curativas para atuação nas fases agudas e de cuidados para as pessoas que perderem a capacidade funcional.

Nesta perspectiva, Camarano (2014, p. 646) ainda ressalta a necessidade de considerar que "os determinantes das condições de saúde vão muito além de um estilo de vida saudável. O Brasil é caracterizado por grandes desigualdades sociais, o que faz com que a sua população experimente trajetórias de vida diferenciadas e, consequentemente, envelheçam de formas diferentes".

A percepção de saúde, entendida como aspecto subjetivo e particular dos indivíduos e sua relação com outros aspectos do cotidiano, merece ser investigada mais profundamente, como também o modo como essas relações podem orientar comportamentos e atitudes, particularmente no grupo populacional de pessoas idosas. Assim, o presente estudo teve por objetivo identificar a prevalência de doenças crônicas não transmissíveis e a condição de saúde auto percebida em idosos que são atendidos pela Estratégia da Saúde da Família de um município da Região do Vale do Rio dos Sinos, RS.

\section{METODOLOGIA}

A metodologia utilizada foi de abordagem quantitativa, sendo o tipo de estudo descritivo de corte transversal. A população do estudo foi composta de idosos, de ambos os sexos que são atendidos pela ESF de um município do Vale do Rio dos Sinos. A amostra foi selecionada por conveniência, procedendo da comunidade, sendo representada por 50 idosos que aceitaram colaborar voluntariamente do estudo.

O ambiente escolhido para a coleta de dados foi a Unidade Básica de Saúde onde os idosos são atendidos pela ESF, e o centro comunitário do próprio bairro no qual a ESF atua. Para a captação dos dados, foi aplicado um questionário elaborado pelos pesquisadores contendo questões fechadas relativas a variáveis como idade e sexo, bem como dados referentes à prevalência de doenças crônicas não transmissíveis como hipertensão arterial, dislipidemia, diabetes mellitus, acidente vascular cerebral (AVC), doença pulmonar obstrutiva crônica (DPOC), neoplasia maligna, artrite, reumatismo, osteopenia e osteoporose.

Para a avaliação da condição de saúde auto percebida foi utilizada uma escala de cinco pontos que permitiram a avaliação dos sujeitos segundo o critério subjetivo de percepção da sua própria saúde. A escala utilizada foi sugerida por SABE (Saúde, Bem-estar e Envelhecimento), em estudo realizado por Lebrão e Laurenti (2005).

Utilizou-se a estatística descritiva para apresentar os resultados através das distribuições de frequências absolutas (n) e relativas (\%), valores mínimos e máximos, médias aritméticas e seus respectivos desvios-padrão.

O estudo foi submetido à avaliação do Comitê de Ética em Pesquisa - CEP Feevale. O parecer geral do projeto protocolado foi de aprovação, sendo o número do processo: 4.09.02.11.2136. 


\section{APRESENTAÇÃO E DISCUSSÃO DOS RESULTADOS}

Participaram da pesquisa 33 mulheres e 17 homens, sendo $66 \%$ e $34 \%$ da população respectivamente. No âmbito investigado houve maior procura de mulheres nos serviços de saúde bem como nos centros comunitários. Tal ocorrência pode estar associada ao fenômeno da feminização da velhice, marcado por diferenças no declínio físico e metabólico de homens e mulheres, caracterizando maior longevidade dessas em relação aos homens, que tem sido atribuído à menor exposição a determinados fatores de risco, relacionados ao ambiente de trabalho, menor prevalência de tabagismo e uso de álcool, bem como diferenças quanto à atitude em relação a doenças e incapacidades (MAZO; LOPES; BENEDETTI, 2009).

Dentre os resultados deste estudo destacam-se percentuais semelhantes com predominância do sexo feminino nos achados de Silveira, Kac e Barbosa (2009) realizado com 596 idosos, representando 59,4\%. O estudo de Pilger, Menon e Mathias (2011) com percentual 64,4\% e estudos de Borges et al. (2014) 63,13\%, com predomínio na faixa etária dos 60-69 anos de idade $(51,82 \%)$.

No que refere a distribuição por faixa etária, 10\% estão na faixa de 80 anos ou mais, $38 \%$ entre 70 e 79 anos e $52 \%$ de idosos encontram-se na faixa de 60 a 69 anos, sendo esta considerada a faixa de idosos que mais cresce no país. Dados do IBGE (2008) apresentam percentuais semelhantes ao deste estudo, correspondendo a 57,4\% de indivíduos entre 60 - 70 anos, 30,1\% entre 70 - 80 anos e 12,6\% acima de 80 anos. Levantamento do IBGE (2016) complementa os achados, no que refere ao aumento da expectativa de vida, fenômeno caracterizado pelo aumento do percentual de pessoas com 60 anos ou mais no período de 2005 e 2015, sendo que neste período os idosos passaram de $9,8 \%$ para $14,3 \%$ da população brasileira. Este crescimento se deu em todos os grupos etários de idosos (de 60 a 64 anos, de 65 a 69 anos, de 70 a 74 anos, de 75 a 79 anos e de 80 anos ou mais). Os maiores percentuais de idosos foram encontrados nas Regiões Sul e Sudeste, com 15,9\% e 15,6\%, respectivamente, e o menor na Região Norte, com $10,1 \%$ da população composta por pessoas com 60 anos ou mais de idade.

No que refere ao município estudado, considera-se importante destacar que o percentual total de idosos é de 9,72\%. E o número de idosos na faixa de 60 a 69 anos é de 3394, compondo 58,1\% de idosos do município (IBGE, 2010).

Na sequência serão apresentados os resultados da avaliação da auto percepção da saúde e suas variáveis categóricas relacionadas a auto percepção da Saúde, auto percepção da saúde comparada a outras pessoas da mesma idade e auto percepção da saúde comparada a doze meses anteriores (Tabela $1)$.

Tabela 1 - Avaliação da auto percepção da saúde (n=50)

\begin{tabular}{|c|c|c|c|}
\hline Variável & Categorias & $\mathbf{N}($ total) & $\%$ \\
\hline \multirow{5}{*}{ Auto percepção Saúde } & Excelente & 1 & 2,0 \\
\hline & Muito Boa & 9 & 18,0 \\
\hline & Boa & 26 & 52,0 \\
\hline & Regular & 13 & 26,0 \\
\hline & Ruim & 1 & 2,0 \\
\hline \multirow{5}{*}{ Auto percepção Saúde Comparada Outros } & Excelente & 2 & 4,0 \\
\hline & Muito Boa & 13 & 26,0 \\
\hline & Boa & 26 & 52,0 \\
\hline & Regular & 8 & 16,0 \\
\hline & Ruim & 1 & 2,0 \\
\hline \multirow{3}{*}{ Auto percepção Saúde Comparada 12 meses } & Melhor & 17 & 34,0 \\
\hline & Igual & 24 & 48,0 \\
\hline & Pior & 9 & 18,0 \\
\hline
\end{tabular}

Fonte: Os autores 
A auto percepção de saúde vem sendo utilizada em vários estudos (LEBRÃO; LAURENTI, 2005; CAETANO; IOZZI; CARNEIRO, 2006; BRASIL, 2011b), e esta auto avaliação do estado de saúde é considerada um indicador válido e relevante do estado de saúde de sujeitos e de populações (BRASIL, 2011b). Os autores ainda revelam que a percepção de saúde manifesta ser um importante indicador de mortalidade: pessoas com pior percepção do estado de saúde têm maior risco de morte (por todas as causas) em comparação com as que relatam saúde excelente. Além de preditor da mortalidade (ALVES; RODRIGUES, 2005; BRASIL, 2011b; SILVA; MENEZES, 2007), a percepção da saúde, ou auto avaliação da saúde, também está relacionada ao declínio funcional, sendo utilizada em pesquisas gerontológicas.

Analisando os resultados sobre a percepção de saúde autorelatada dos sujeitos do estudo pode-se destacar que $52 \%$ considera sua saúde boa, $18 \%$ muito boa e $2 \%$ excelente. Considerando essas três alternativas como percepção de saúde positiva, compreende-se que $72 \%$ da população acredita estar bem e demonstra ter cuidado com sua saúde. Tendo em vista o conceito de saúde defendido por Araújo e Xavier (2014) em seu sentido mais amplo, "saúde como defesa da vida, situada como um estado que pode ser promovido, buscado, cultivado e aperfeiçoado", obteve-se nesta pesquisa um índice elevado de satisfação com a sua própria saúde.

Dados que corroboram os achados do presente estudo foram encontrados no estudo realizado por Pilger, Menon e Mathias (2011), no qual a auto percepção da saúde atual foi considerada boa por 54,8\% da população idosa estudada. Da mesma forma, no estudo de Borges et al. (2014) grande parte dos idosos considerou sua saúde como boa $(47,81 \%)$.

Analisando a variável autopercepção de saúde comparada a outras pessoas da mesma idade, os resultados obtidos na pesquisa foram de $52 \%$ para a categoria boa, igualando ao resultado da primeira variável de percepção de saúde autorelatada. Somando-se os resultados positivos, obtem-se $82 \%$ de satisfação com a saúde e somente $18 \%$ de insatisfação comparada a outros sujeitos.

Quando os idosos responderam em relação a sua saúde comparada a de doze meses atrás, $48 \%$ considerou sua saúde igual, muito próximo ao estudo de Fernandes et al. (2010), que obteve 45\%. Em relação as outras duas categorias, inverteram-se os resultados, na pesquisa em questão, uma vez que a categoria melhor apresentou $34 \%$ das escolhas, e a categoria pior 18\%, enquanto que na pesquisa de Fernandes et al. (2010), foram 30\% para pior e 25\% para melhor. Quando avaliada a sua saúde no estudo de Ebert (2012) os idosos, mostram num percentual de 39,5\% que é boa, 25,3\% que ela é regular e 4,3\% avaliam como muito ruim. Na avaliação do cuidado com a sua saúde, $15 \%$ avalia como excelente $30,9 \%$ como muito boa, e $37,7 \%$ como boa. Observa-se a partir das respostas uma positividade na maneira de avaliar a saúde entre os idosos em ambos os estudos.

Tabela 2 - Fatores de risco associados à saúde $(\mathbf{n = 5 0})$

\begin{tabular}{|c|c|c|c|}
\hline Variável & Categorias & $\mathbf{N}$ & $\%$ \\
\hline \multirow[t]{9}{*}{ Doenças Crônicas NT } & HAS & 32 & 64,0 \\
\hline & Artrite / Reumatismo & 18 & 36,0 \\
\hline & Dislipidemia & 17 & 34,0 \\
\hline & Diabetes Mellitus & 14 & 28,0 \\
\hline & Osteopenia / Osteoporose & 7 & 14,0 \\
\hline & Neoplasia Maligna & 6 & 12,0 \\
\hline & AVC & 5 & 10,0 \\
\hline & DPOC & 5 & 10,0 \\
\hline & Nenhuma & 2 & 4,0 \\
\hline
\end{tabular}

Fonte: Os autores 
Observando a tabela 2 podemos destacar que a Hipertensão Arterial Sistêmica é a doença que mais acomete os idosos da pesquisa, isto é $64 \%$ declararam ter a enfermidade, logo, com $36 \%$ as doenças relacionadas ao reumatismo, como artrite/artrose. Em seguida a doença mais citada foi a dislipidemia com $34 \%$ e abaixo dela a diabetes mellitus com $28 \%$. Dados que indicam conformidade com a prevalência de HAS no Brasil, informados nos Arquivos Brasileiros de Cardiologia, atingem 32,5\% (36 milhões) de indivíduos adultos, mais de 60\% dos idosos, contribuindo direta ou indiretamente para 50\% das mortes por doença cardiovascular (MALACHIAS et al., 2016).

Perante esta prevalência, considera-se importante fazer referência a estudos importantes como de Alves, Leite e Machado (2010), Barcelos e Oliveira (2011) e Folmann (2011) que revelam a HAS como a doença crônica mais frequente. Em estudo realizado por Ebert (2012) as Doenças cardiovasculares alcançaram $24,8 \%$ e a HAS $68,5 \%$, atingindo a grande maioria dos idosos da amostra, seguidas das doenças articulares-reumatismo-osteoporose (57,6\%). Quanto às características clínicas dos idosos, percebemos que a HAS, atinge a grande maioria dos idosos, seguida das doenças articulares. Portanto, esta prevalência caracteriza uma das causas de maior redução da qualidade e expectativa de vida desta população.

Os agravos decorrentes das DCNT têm sido as principais causas de morte na população idosa. Quando são analisadas as causas especificas, a doença cerebrovascular ocupa o primeiro lugar em mortalidade do país, tanto em idosos como na população geral. E em segundo lugar estão as doenças cardiovasculares. Um importante motivo do Brasil alcançar um número tão elevado de indivíduos acometidos pela doença cerebrovascular é a alta prevalência de hipertensão arterial da população brasileira e o não tratamento, ou o tratamento inadequado dessa enfermidade (BRASIL, 2010).

Os resultados obtidos em relação a morbidades associadas ao sistema osteoarticular como as doenças reumatológicas, artrite, reumatismo e artrose, juntas somaram 36\%. Diante deste contexto, considera-se importante evidenciar que estas doenças crônicas constituem uma ameaça à autonomia do idoso, associada a dor e incapacidade funcional, afetando o bem-estar geral desses indivíduos quando portadores de algumas dessas doenças.

A Diabete Mellitus também é uma doença importante no cenário das DCNT, obteve um resultado de $28 \%$ de prevalência no estudo. Folmann (2011) também obteve resultado aproximado $(24,43 \%)$. A pesquisa ainda evidenciou outras DCNT, a osteopenia, osteoporose, neoplasia maligna, AVC e DPOC, que obtiveram um percentual menor de prevalência.

Hipertensão, diabetes e artrite/artrose foram também as doenças crônicas mais prevalentes encontradas no estudo de Pilger, Menon e Mathias (2011), confirmando observações previamente realizadas em outros estudos da população idosa. A frequência da hipertensão autorreferida foi de 30,5\% entre os homens e $37,1 \%$ entre as mulheres e representou 34,9\% do total das doenças relatadas.

Considera-se ainda importante fazer referência aos dados do Sistema de Informações sobre Mortalidade - SIM, fornecidos pelo Ministério da Saúde, relativo a distribuição percentual de óbitos, por grupos de causas definidas, segundo os grupos de idade - Brasil - 2014, constante na síntese de indicadores sociais do IBGE (2016) no qual a mortalidade dos idosos está mais concentrada nas doenças do aparelho circulatório (36,3\%), seguida pelas neoplasias $(18,6 \%)$ e doenças do aparelho respiratório $(15,5 \%)$. É importante mencionar que as mortes por causas não definidas correspondiam a 5,8\% do total dos óbitos em 2014.

Diante dos resultados encontrados neste estudo, interligado a estimativa que o Brasil tem uma das populações que envelhecem mais rapidamente no mundo, a carga de doenças crônicas no país tende a aumentar, exigindo um novo modelo de atenção à saúde para essa população (BRASIL, 2011c). 


\section{CONSIDERAÇÕES FINAIS}

O estudo contou com um total de 50 idosos, com idade média de 69 anos. As mulheres representaram $66 \%$ da amostra, caracterizando a feminização do envelhecimento, já evidenciada no corpo do trabalho.

Em relação à auto percepção de saúde, o estudo apresentou resultados positivos, mesmo sendo constatada a prevalência de várias doenças crônicas não transmissíveis. Dado esse que evidencia a subjetividade da percepção de saúde, associada a inúmeros fatores, dentre eles, história de vida, doenças superadas, autoestima, ambiente no qual vivem.

A auto percepção de saúde dos idosos pode ser usada como ferramenta para melhorar as condições de saúde e acesso aos serviços de saúde por parte desta população, independentemente da condição social. Assim, ações que abordam os principais fatores determinantes da auto percepção de saúde podem contribuir de maneira significativa para a promoção da saúde, bem-estar e qualidade de vida dos idosos.

\section{REFERÊNCIAS}

ALVES, L. C.; LEITE, I. C.; MACHADO, C. J. Fatores associados á incapacidade funcional dos idosos no Brasil: análise multinível. Revista Saúde Pública, v. 44, n. 3, p. 468-78, 2010. Disponível em: 〈http://www.scielosp.org/pdf/rsp/v44n3/10.pdf>. Acesso em: 03 abr. 2017.

ALVES, L. C.; RODRIGUES, R. N. Determinantes da autopercepção de saúde entre idosos do Município de São Paulo, Brasil. Rev. Panam. Salud Publica, v. 17, n. 5/6, p. 333-41, 2005. Disponível em: <http://www.scielosp.org/pdf/rpsp/v17n5-6/26270.pdf Acesso em abril 2012>. Acesso em: 19 abr. 2017.

ARAÚJO, J. S.; XAVIER, M. P. O conceito de saúde e os modelos de assistência: considerações e perspectivas em mudança. Revista Saúde em Foco, Teresina, v. 1, n. 1, art. 10, p. 117-149, jan./jul. 2014. Disponível em:

<http://www4.fsanet.com.br/revista/index.php/saudeemfoco/article/viewFile/326/382\&gws_rd=cr\&ei =ycB-WJeGNMebwASnrrrwAg>. Acesso em: 08 mai. 2017.

BARCELOS, A. R. G.; OLIVEIRA, L. I. E. Relações Entre Autopercepção de Saúde, Aspectos Sociodemográficos e Doenças Crônicas Não Transmissíveis de Idosas que Frequentam um Projeto Social de uma Cidade do Vale do Rio dos Sinos-RS. Revista Conhecimento Online, v. 3, n. 2, 2011. Disponível em: <http://www.feevale.br/hotsite/default.asp?intIdHotSite=86\&intIdSecao=5265\&>. Acesso em: 03 abr. 2017.

BORGES, A. M. et al. Autopercepção de saúde em idosos residentes em um município do interior do Rio Grande do Sul. Rev. Bras. Geriatr. Gerontol., Rio de Janeiro, v. 17, n. 1, p. 79-86, 2014; Disponível em: <http://www.scielo.br/pdf/rbgg/v17n1/1809-9823-rbgg-17-01-00079.pdf>. Acesso em: 19 abr. 2017.

BRASIL. MINISTÉRIO DA SAÚDE. Atenção à Saúde da Pessoa Idosa e Envelhecimento. Série Pactos pela Saúde 2006, vol.12. Brasília. DF. 2010. Disponível em: <http://portal.saude.gov.br/portal/arquivos/pdf/volume12.pdf>. Acesso em: 21 mar. 2017.

BRASIL. MINISTÉRIO DA SAÚDE. Proposta de capacitação em saúde do idoso. Saúde do idoso. 2011a. Disponível em <www.saude.gov.br/programas/idoso/proposta.htm>. Acesso em: 21 mar. 2017. 
BRASIL. MINISTÉRIO DA SAÚDE. VIGITEL BRASIL 2010, Vigilância de Fatores de Risco e Proteção para Doenças Crônicas por Inquérito Telefônico: Estimativas sobre frequência e distribuição sociodemográfica de fatores de risco e proteção para doenças crônicas nas capitais dos 26 Estados Brasileiros e Distrito Federal em 2010. Série G. Estatística e Informação em Saúde. Brasília DF, 2011b. Disponível em:

<http://portal.saude.gov.br/portal/arquivos/pdf/vigitel_2010_preliminar_web.pdf >. Acesso em: 21 mar. 2017.

BRASIL. MINISTÉRIO DA SAÚDE. Secretaria de Vigilância em Saúde. Departamento de Análise de Situação de Saúde. Plano de ações estratégicas para o enfrentamento das doenças crônicas não transmissíveis (DCNT) no Brasil 2011-2022 / Ministério da Saúde. Secretaria de Vigilância em Saúde. Departamento de Análise de Situação de Saúde. - Brasília : Ministério da Saúde, 2011c. 160 p. Disponível em: <http://bvsms.saude.gov.br/bvs/publicacoes/plano_acoes_enfrent_dcnt_2011.pdf>. Acesso em: 08 mai. 2017.

CAETANO, S. C.; IOZZI, R.; CARNEIRO, A. Percepção do Estado de Saúde do Idoso na Cidade do Rio de Janeiro, 2006. Trabalho apresentado no XVI Encontro Nacional de Estudos Populacionais, realizado em Caxambu MG Brasil, de 29 de setembro a 03 de outubro de 2008. Disponível em: <http://abep.nepo.unicamp.br/encontro2008/docspdf/ABEP2008_1194.pdf>. Acesso em: 21 mar. 2017.

CAMARANO, A. A. (Org.). Novo regime demográfico: uma nova relação entre população e desenvolvimento? In: CAMARANO, A. A. Novo regime demográfico: uma nova relação entre população e desenvolvimento? Rio de Janeiro: Ipea, 2014, p. 627-654.

EBERT, N. H. Doenças crônicas, fragilidade e características emocionais de idosos comunitários: estudo fibra Ivoti/RS, 2012. 116 f. Dissertação (Mestrado em Inclusão Social e Acessibilidade) Feevale, Novo Hamburgo-RS, 2012.

FERNANDES, A. J. F.; PIRES, C. G.; RIBEIRO, S. L.; MAAGH, S. B.; MIRAPALHETA, P.; LANGE, C. Autopercepção de saúde de idosos vítimas de causas externas atendidos no Pronto Socorro de Pelotas. In: Anais... Congresso de Iniciação Científica, 2010, Pelotas. II Mostra Científica, 2010.

FOLMANN, L. Estado nutricional dos idosos usuários de uma Unidade Básica de Saúde em Porto Alegre - RS. Porto Alegre, novembro de 2011. TCC Nutrição UFRGS. 2011 Disponível em: <http://hdl.handle.net/10183/37214>. Acesso em: 21 mar. 2017.

FREITAS, M. C.; QUEIROZ, T. A.; SOUSA, J. A. V. O significado da velhice e da experiência de envelhecer para os idosos. Rev. Esc. Enferm. USP, São Paulo (SP), v. 44, n. 2, p. 407-12, 2010. Disponível em: <http://www.scielo.br/pdf/reeusp/v44n2/24.pdf>. Acesso em: 08 mai. 2017.

IBGE. Projeção da População do Brasil por sexo e idade: 1980-2050. Rio de Janeiro: IBGE, 2008. Disponível em: <http://www.ibge.gov.br/home/estatistica/populacao/projecao_da_ populacao/2008/default.shtm>. Acesso em: 21 mar. 2017.

IBGE. Cidades @. Dados do Censo 2010 e extensão territorial dos municípios. Rio de Janeiro: IBGE, 2010. Disponível em: <www.ibge.gov.br/cidadesat/topwindow.htm?1>. Acesso em: 21 mar. 2017.

IBGE. Síntese de indicadores sociais: uma análise das condições de vida da população brasileira: 2016/IBGE, Coordenação de População e Indicadores Sociais. Rio de Janeiro: IBGE, 2016. 146 p. Disponível em: <http://biblioteca.ibge.gov.br/visualizacao/livros/liv98965.pdf>. Acesso em: 21 mar. 2017. 
LEBRÃO, M. L.; LAURENTI, R. Saúde, bem-estar e envelhecimento: o estudo SABE no município de São Paulo. Rev. Bras. Epidemiologia, 2005, v. 8, n. 2, p. 127-41. Disponível em:

<http://www.scielosp.org/pdf/rbepid/v8n2/05.pdf>. Acesso em: 19 abr. 2017.

MALACHIAS, M.V.B.; SOUZA, W.K.S.B.; PLAVNIK, F. L.; RODRIGUES, C.I.S.; BRANDÃO, A.A.; NEVES, M.F.T. et al. $7^{\text {a }}$ Diretriz Brasileira de Hipertensão Arterial. Arquivo Brasileiro de Cardiologia 2016; 107(3 Supl.3):1-83. Disponível em:

<http://publicacoes.cardiol.br/2014/diretrizes/2016/05_HIPERTENSAO_ARTERIAL.pdf >. Acesso em: 10 mai. 2017.

MAZO, G. Z.; LOPES, M. A.; BENEDETTI, T. B. Atividade Física e o Idoso: Concepção Gerontológica. 3. ed. revisada e ampliada. Porto Alegre: Sulina, 2009. 318 p.

PILGER, C.; MENON, M. H.; MATHIAS, T. A. F. Características sociodemográficas e de saúde de idosos: contribuições para os serviços de saúde. Rev Latinoam Enferm, 2011. v. 19, n. 5, p. 1-9. Disponível em: <http://www.scielo.br/pdf/rlae/v19n5/pt_22.pdf>. Acesso em: 19 abr. 2017.

PITANGA, F. J. G. Epidemiologia: da Atividade Física, do Exercício Físico e da Saúde. 3 ed. São Paulo: Phorte editora, 2010.

SANTOS, S. S. C. Concepções teórico-filosóficas sobre gerontogeriátrica envelhecimento, velhice, idoso e enfermagem gerontogeriátrica. Rev. Bras. Enferm., Brasília (DF), v. 63, n. 6, p. 1035-9, novdez, 2010. Disponível em: 〈http://www.scielo.br/pdf/reben/v63n6/25.pdf>. Acesso em: 08 mai. 2017.

SILVA, T. R.; MENEZES, P. R. Autopercepção de saúde: um estudo com idosos de baixa renda de São Paulo. Rev. Med. São Paulo., v. 86, n. 1, p. 28-38, 2007. Disponível em: <http://medicina.fm.usp.br/gdc/docs/revistadc_96_p.28-38\%20861pdf〉. Acesso em: 19 abr. 2017.

SILVEIRA, E. A.; KAC, G.; BARBOSA, L. S. Prevalência e fatores associados à obesidade em idosos residentes em Pelotas, Rio Grande do Sul, Brasil: classificação da obesidade segundo dois pontos de corte do índice de massa corporal. Cad. Saúde Pública, Rio de Janeiro, v. 25, n. 7, p. 15691577, jul 2009. Disponível em: 〈http://scielo.br/pdf/csp/v25n7/15.pdf〉. Acesso em: 19 abr. 2017.

SOUZA, M. A. C. Esporte Para Idosos: Uma abordagem inclusiva. São Paulo: SESCSP, 2010.

TERRA, N.; OPPERMANN, R.; TERRA, P. Doenças Geriátricas e Exercícios Físicos. Porto Alegre: ediPUCRS, 2010. 